\title{
Study of Histological changes in placenta of anaemic mothers .
}

\author{
Dr. Ritu Bala Soni ${ }^{1}$, Dr. Shema Nair ${ }^{2}$ \\ 1. Assistant Professor, Department Of Anatomy, Gandhi Medical college, Bhopal .( M.P.) India \\ 2. Assistant Professor, Department Of Anatomy, L.N. Medical College, Bhopal. (M.P.) India
}

\begin{abstract}
Anaemia in female during pregnancy is very common, it affects maternal blood, leading to hypoxia, which cause changes in structure of Placenta. Aanemia also exert profound changes on the maternal circulatory system and has serious effects both on mother and fetus. Growth of the fetus is intricately linked with that of placenta, because of transfer of nutrients and oxygen through placenta. Microscopic study revealed histomorphological abnormalities due to hypoxia comprise of some changes in structure of placenta like increased capillaries per villous, thickening of capillary basement membrane and trophoblastic basement membrane , thickening of of syncytiotrophoblast, increased fibrosis and syncytial knots and appearance of vasculosyncytial membrane. All these changes occurred for maintaining sufficient efficiency of placenta due to compensatory phenomena, for fulfill demand of oxygen in the fetus .
\end{abstract}

Key Words: Anaemia, hypoxia, placenta.

\section{Introduction}

Placenta is a fountainhead of human existence because it is a means for provide nutrition and exchange of gases (o $2 \&$ co2 ) in fetus . So it is a vital organ which is absolutely essential for survival, growth and development of fetus.

In placenta maternal blood circulate through the intervillous space and fetal blood circulate through blood vessels in the villous space. Villi are functional unit of placenta .In placenta maternal blood and fetal blood do not mix with each other .

The structure across which transport occurs are collectively known as placental barrier. In human placenta barrier is composed entirely of tissues of fetal origin ; these are A) endothelium of fetal blood vessels and its basement membrane , B) surrounding mesoderm , C ) cytotrophoblast and its basement membrane, D ) syncytiotrophoblast .

Total area of this membrane is as great as 14 square meter (Equal to total absorptive area of the adult intestinal tract ).

Any diseases either associated or aggravates with pregnancy like hematological disorder, diabetes and hypertension affects placenta, which also affected morbidity and mortality statistics of pregnancy and fetal outcome .

Anaemia is commonest hematological disorder that occurs in pregnancy . Severity of anaemia among expectant mothers was judged by criteria suggested by WHO[1] and according to which a level of haemoglobin below $11 \mathrm{gm} / \mathrm{dl}$ during pregnancy is an indication of anaemia .

The commonest cause of anaemia during pregnancy is iron deficiency which may be due to nutritional deficiency or increased demand of oxygen in pregnancy. Anaemia leading to hypoxia cause changes in structure of Placenta .Anaemia also exerts profound changes on the maternal circulatory system and has serious effects both on mother and fetus .

It was observed by G.H. Burton et al [2] that fetal vasculature of human placenta adapt in a uniform manner in different form of hypoxic stress like high altitude and maternal iron deficiency anaemia . Increased capillarization of term villi is found at high altitude and with some other hypoxic condition like maternal iron deficiency anaemia .Dilatation of capillary sinusoid with accompanying thinning of the villous membrane is the principal adaptation to hypoxia . Due to this sinusoidal dilatation of terminal villous capillaries vasculosyncytial membrane form . This membrane bulge against the trophoblastic surfaces. The factors responsible for the formation of stromal fibrosis are normal ageing process and reduced uteroplacental blood flow . It is fibrin which is formed by thrombosis of maternal blood in intervillous space. Thrombosis occurs as a result of eddy currents; this decreases the intervillous space. Fibrin deposition causes reduction in the villous surface area .

The syncytiotrophoblast of entrapped villi degenerates and the stroma of the villi becomes markedly fibrotic. With this the villous population decreases further and the nutritional demands of the fetus are not adequately fulfilled. Stromal fibrosis was seen in $13 \%$ in anaemic cases as compared to $7 \%$ in normal cases . Because in anaemia there is a relative hypoxia, which may be the main factor for its production .

Low oxygen causes cytotrophoblastic proliferation and leading to increase in syncytium thickness.. 
Charnock Jones D [3] , Kaurfmann P[4] (2004) stated that fetoplacental angiogenesis vary in certain pregnancy pathologies. Changes are seen in hypoxia in vascular endothelium, total capillary growth, villous capillarization and capillary size and shape.

In mature villi, in syncytotrpphoblast ( synctium) nuclei are irregularly dispersed and often appear aggregated to form multinucleated protrusions from the villous surface known as syncytial knots . Syncytial knots represent degenerated nuclei . Frequently the syncytium becomes very thin and syncytial knots may break off and drop into intervillous space. Syncytial knots enter in maternal blood circulation and usually degenerate without causing any symptoms but rarely it may cause embolism and leads lung infarction .

\section{Material \& Method}

60 placentas were taken from mothers (Full term) who delivered at hospital constituted the material for present study .Full term placentas were collected along with detailed case history and relevant investigations immediately after delivery of fetus.All deliveries were normal vaginal delivery The placentas were washed with normal saline and preserved in 10\% saline for microscopic examination.

This study was divided into 2 groups -

Group I (control group)- comprised of 30 placentas from mothers having no sign and symptoms of anaemia and their hemoglobin level were recorded to be more than $11 \mathrm{gm} \%$.

GROUP II (Study group )- comprised of 30 placentas from mothers having anaemia (hemoglobin level $<11 \mathrm{gm}$ $\%)$.According to WHO report [5] study group redivided into 3 groups depending on the severity of anaemia .

Group IIA - Mild Anemia (Haemoglobin level $11-10 \mathrm{gm} / \mathrm{dl}$ )

Group IIB - Moderate Anemia (Haemoglobin level $10-7 \mathrm{gm} / \mathrm{dl}$ )

Group IIC - Severe Anemia (Haemoglobin level $<7 \mathrm{gm} / \mathrm{dl}$ )

$2 \mathrm{~cm}$ pieces were removed from both group of placenta included both fetal and maternal surface. Pieces are fixed in $10 \%$ formalin for 24 hours then $2 \mathrm{~mm}$ section were taken (Through and through) and further used for processing and staining .

\section{Method of processing}

1. Dehydration - The preserved tissues washed in running tap water for 4-6 minutes . They were then passed through upgraded alcohol as follows-

$50 \%$ alcohal - 1 hour

$70 \%$ alcohal - 1 hour

$90 \%$ alcohal - 1 hour

Absolute alcohal I- 1hour

Absolute alcohal II - 1hour

2 . Clearing - Clearing of tissue done in xylene, 1hour in xylene I , then after in xylene II .

3 .Embedding -

The cleared tissue was put in molten wax(melting point $56^{\circ}$ celsius $-58^{0}$ celsius ) for 12 hours in oven . The paraffin blocks of tissue were made with help of Lockhart, s metallic blocks .

4 . Sectioning - The serial paraffin sections of 5 micron thickness were cut by rotator microtome and floated in water bath having temperature 45-50 degree Celsius. The section were made spread on the slide smeared with adhesive solution (mixture of equal amount of glycerol and egg albumin). The slide was dried on hot plate having temperature 50 degree celcius.

5 . Deparaffinisation of sections - The slide was put in xylene , 2 changes each for 5-10 min in order to remove the extracellular and intracellular wax .

6 . Rehydration - The slide were put in descending grades of alcohol i.e absolute $90 \%, 70 \%$ and $50 \%$ alcohol for $2 \mathrm{~min}$ each. The slide was then washed in running tap water for 2 minute and then taken for routine H \& E staining.

\section{Method Of Staining}

1-Stained with Harris haematoxylene for 7-8 min.

2 - Washed in running tap water until section become blue .

3 - Then section observed by microscope ; if section is understained then put again in haematoxylene for 2-3

min. If section is overstained then differentiate in $1 \%$ acid alcohol ( $1 \% \mathrm{HCL}$ in $70 \%$ alcohal) for $5-10 \mathrm{sec}$.

4 - Stained in $1 \%$ eosin for $7-10 \mathrm{~min}$.

5 - Washed in running tap water (1-2min )

6 -Dehydrated through

Alcohol I (75 \%) - For 1 min

Alcohol II ( Absolute alcohal) 1 -2 dip .

7- Cleaned by - Xylene I - 2dip 
Xylene II - 2dip

8 - Mounting - By DPX

Observation- microscopic examination were done under following headings -

1 ) Capillaries per villous- In control group each villous had minimal number of small capillaries but in study group Capillaries per villi were seen to be increased in number and dilated with severity of anaemia.

2 ) Cytotrophoblastic proliferation (more than one layer of cytotrpphoblast in villi ) - In control group we did not found any case of cytotrophoblastic proliferation, whereas in study group cytotrophoblastic proliferation increases with severity of anaemia .

3 ) Thickening of Basememt Membrane of villous capillaries - In control group basement membrane of villous capillaries was observed to be normal whereas it is increased in thickness with severity of anaemia .

4 ) Thickening of Basement membrane of trophoblast - In control group basement membrane of trophoblast was observed to be normal whereas it is increased in thickness with severity of anaemia .

5 ) Vasculosyncytial membrane -

(Sinusoidal dilatation of terminal villus capillaries form vasculosyncytial membrane.)

Cases of Vasculosyncytial membrane were not found in cases of control group whereas in study group occurrences of Vasculosyncytial membrane increase with increase in severity of anaemia .

6 ) Fibrosis- In control group scattered and minimal amount of fibrosis were seen whereas in study group amount of fibrosis tend to increase in villi and in intervillous spaces with increase in severity of anaemia .

7 ) Syncytial knots - Syncytial knots occasionally seen in cases of control group whereas in study group occurrences of Syncytial knots increase with increase in severity of anaemia .

Photomicrographs of placenta

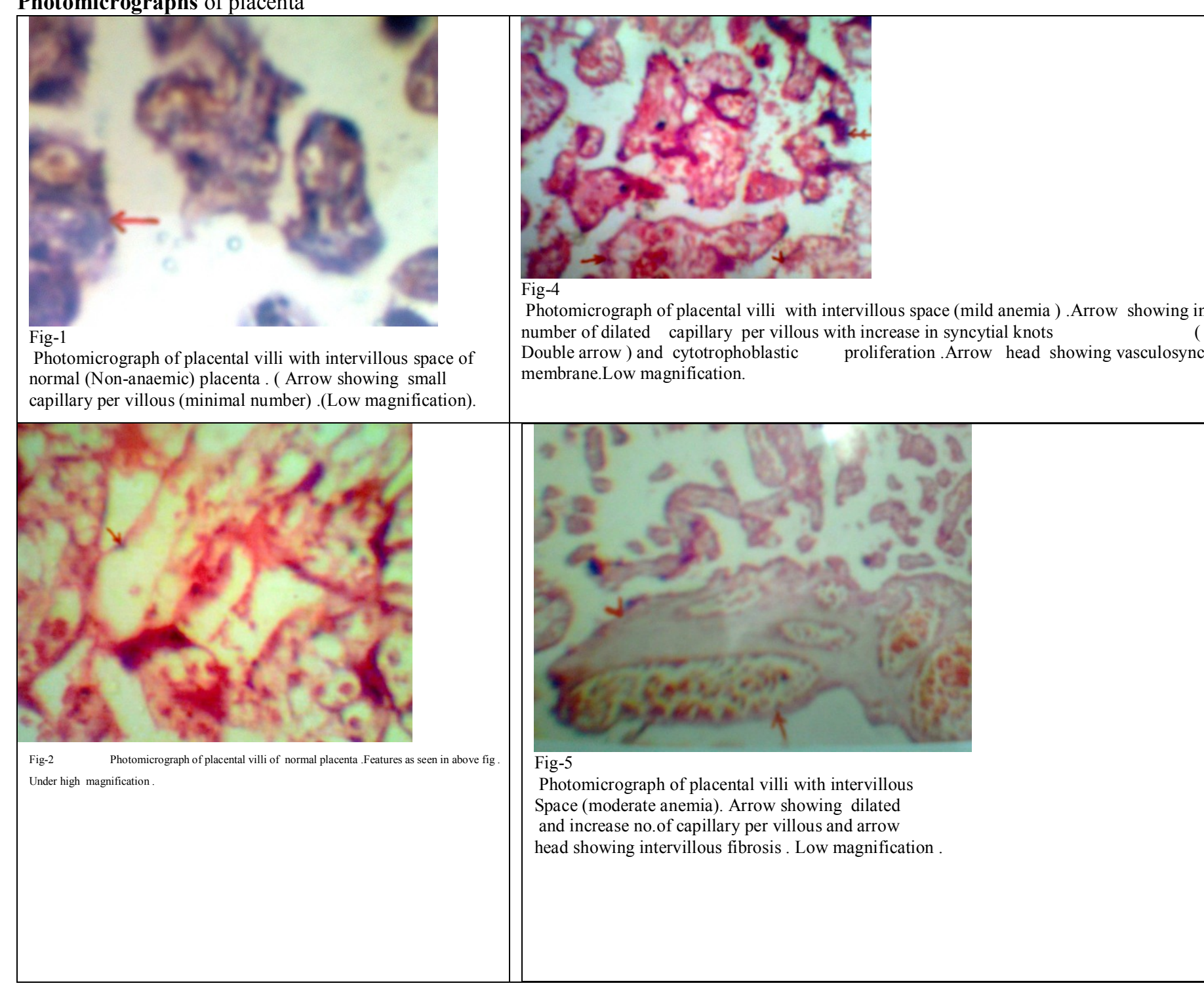



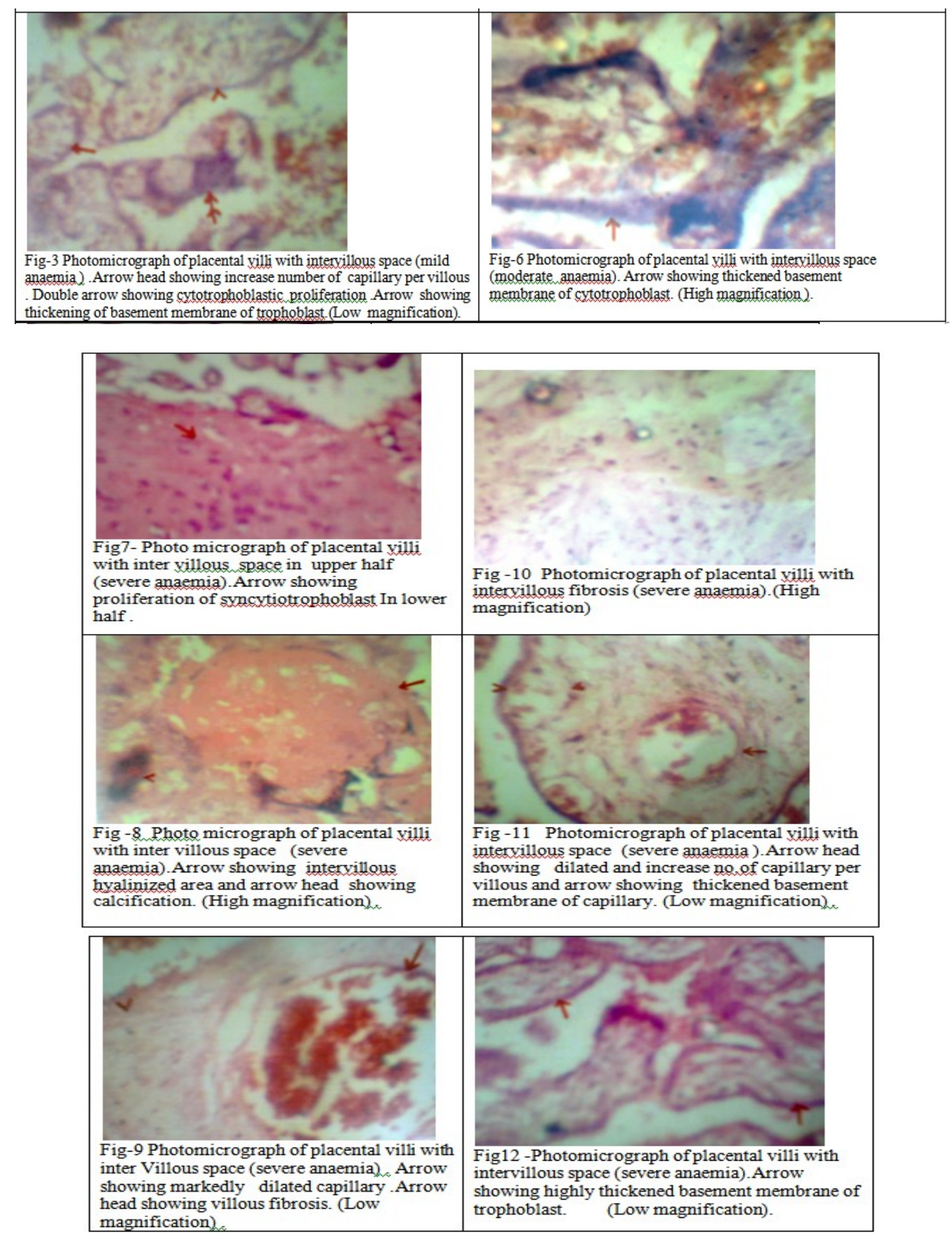

\section{Disscusion \& Conclusion}

In present study it was observed that the capillaries per villous increases in number and are dilated with increasing grade of anaemia . Reshetnikova OS [6], Burton GJ (1995) studied placental histomorphometry of villous membrane in pregnancies complicated by maternal iron deficiency anaemia . Burton stated that raised capillary density and dilatation of sinusoids is the principal adaptation to hypoxia Simpson[7] also reported that there is hypoxic hypercapillarization in ischemic placenta .It is correlated with our study findings.

H . Fox [8] stated that placenta subjected to ischemia show cytotrotrophoblastic cell hyperplasia. Mariana sayeed [9]observed that Cytotrophoblastic proliferation was for replacement of damaged cytotrophoblast caused by ischemia. So Cytotrophoblastic proliferation is a repair phenomenon and it is an 
adaptation to physiological stress. In our study we also found that cytotrophoblastic proliferation present in anaemic placenta which is increases with severity of anaemia. It is correlated with our study findings.

In present study it was observed that vasculosyncytial membrane increases with severity of anemia. This was correlated with study of GetzowA [10] and sodowsky [11] (1950). According to Getzow A and Sodowsky, in some area of villi the syncytium becomes anuclear and attenuated overlying a dilated foetal capillary to form Vasculosyncytial membrane for facilitation of gas transfer across the placenta as they represent the site of closest approximation of maternal and foetal blood circulation . It means formation of Vasculosyncytial membrane is a accommodative effect to face problem of hypoxia. Same was also stated by Fox $\mathbf{H}$ [12] . Because of same reason in our study cases of Vasculosyncytial membrane were not found in cases of control group whereas in study group occurrences of Vasculosyncytial membrane increases with increase in severity of anaemia .

In present study we observed that stromal fibrosis increases with severity of anemia. It was also stated by Usha Russia[13] that villous fibrosis increases in amount with increasing grade of anaemia .

In study group syncytial knots found.According to Jones C \& Fox H [14] formation of syncytial knots indicates ischemic response (under hypoxic condition like maternal anaemia) or functional inactivity

[1]. WHO Report. Technical Report 1989; 776 : p 308-310

\section{Bibliography}

[2]. Burton G .J., Reshetnikova O. S. Milovanov A. P. and Teleshova O. V. Stereological 1 evaluations of vascular adaptation in human Placental villi to differing forms of hypoxic stress. Placenta 1996.

[3] \& [4] Charnock- Jones D S, Kaufmann P. Aspects of Feto-placenta 1 vasculogenesis and angiogenesis. Placenta. 2004 Feb March;

[5] WHO Report of working group on Anaemia. 1992.

[6] Reshetnikova O S, Burton G J. Placental histomorphometry and morphmetric diffusing capacity of the villous membrane in pregnancies complicated by maternal iron-deficiency anaemia. . AMJ Obstectrics \&. Gynecology. 1995 Sep;

[7] Simpson R.A., Mayhew T.M. \& Barnes P. R. Histopathological approach to villous alterations. Placenta 2003.

[8] Fox H . The Morphological Basis of Placental Insufficiency. The Journal of Obstetrics and Gynecology of India. and Gynecology of India. Aug. 1975.

[9] Marrinne Sayeed, Chakarwarti R.N. and Devi P. K. A comparative study of placental villous changes in normal and abnormal pregnancies . Journal of obstetrics and Gynecology of India ; September 1974

[10] Getzow AS, Garm S M, Redella SA, Falkner F. Maternal hematological levels and pregnancy out come.1981.

[11] Sadowsky A and Getzowa A.S. . The Structure of the Human Placenta with Full term and Immature Fetus Living or Dead. Journal of Obstetrics Gyneacology; British Common Wealth. 1950.

[12] Fox H. The incidence and significance of Vasculosyential membrane in Human term placenta. Journal of obstrics \& Gynae British common wealth .1967.

[13] Usha Rusia, Arti Bhatia, Suma Kapoor, Nishi Madan, Vijaylaxmi Nair and S .K. Sood. Placental Morphology and Histochemistry in iron deficiency anaemia.. Indian Journal Med Res. May 1998

[14] Jones C \& Fox H .Syncytial knots and intervillous bridges in the Human placenta. An Ultrastructural Study . Journal of Anatomy .1977 